\title{
Influence of Microstructure on Coercive Force of Pure Iron Powder Cores*
}

\author{
Takuya Takashita and Naomichi Nakamura \\ Steel Research Laboratory, JFE Steel Corporation, Chiba 260-0835, Japan
}

The recrystallization behavior of pure iron powder cores during the annealing process is visualized as Grain Orientation Spread (GOS) maps with an SEM/EBSD analysis. It is found to be significantly heterogeneous, leading to a heterogeneous dislocation density distribution. Nevertheless, a conventional dislocation pinning model well describes the coercive force behavior if the average dislocation density is applied. The pinning model is modified to separate the influence of the grain boundary pinning, and the relationship between the coercive force and the microstructure is discussed comprehensively. [doi:10.2320/matertrans.Y-M2018821]

(Received March 28, 2018; Accepted May 2, 2018; Published June 8, 2018)

Keywords: pure iron powder, coercive force, magnetic properties, atomized iron powder, dislocation density, SEM/EBSD

\section{Introduction}

Pure iron powder cores are made from insulation-coated pure iron powders through a powder compaction process, and featured with their saturation magnetic flux densities higher than those of conventional ferrite cores and eddy current losses lower than those of conventional metal magnetic materials such as electrical steel sheets. With these features, iron powder cores have been applied to devices such as reactors for hybrid vehicles. ${ }^{1)}$

To expand the application field of pure iron powder cores, reduction of the coercive force is required. Defects such as inclusions, precipitate particles, crystal grain boundaries and dislocations act as domain wall pinning sites during the magnetization process. $^{2)}$ Therefore, quantitative understanding of the influence of such pinning sites is important for the reduction of the coercive force.

Several studies on the coercive force of pure iron powder cores have been reported. Takashita et $a l .{ }^{3)}$ have reported that the coercive force of a pure iron powder core shows a linear relationship with the inverse of the crystal grain size, and that coercive force decreases with an increase in the crystal grain size. Moreover, crystal grain size in a pure iron powder core after annealing increases with an increase in the circularity of the base iron powder because of a decrease in the dislocation density induced during compaction. Nishi et al. ${ }^{4}$ ) have reported the influence of oxide particles on the coercive force of pure iron powder cores, and concluded that coercive force increases with an increase in the number of oxide particles per unit volume.

For polycrystalline bulk iron, Yaegashi et al. ${ }^{5)}$ have found that the coercive force is proportional to the $1 / 2$ power of the dislocation density.

However, neither of those studies have separated the influence of dislocations from the domain wall pinning sites.

In this study, the influence of the dislocation density on the coercive force of pure iron powder cores is evaluated separately from the microstructural factors such as grain size and inclusions.

\footnotetext{
*This Paper was Originally Published in Japanese in J. Jpn Soc. Powder Powder Metallurgy 64 (2017) 428-435. In order to more precisely explain the relationship between annealing temperature and area fraction of recrystallized grain, Fig. 11 was changed.
}

\section{Experiment}

\subsection{Preparation of iron powder cores}

The iron powder cores were fabricated through the process shown in Fig. 1. The as-atomized iron powder was annealed at $1223 \mathrm{~K}$ for $3.6 \mathrm{ks}$ in hydrogen atmosphere, and sieved and remixed into a base iron powder with the apparent density and particle size distribution in Table 1. The chemical composition of the base iron powder is shown in Table 2.

The base iron powder was mixed with a silicon resin solution in which the silicon resin (Dow Corning Toray, SR2400) was diluted to 1 mass $\%$ with xylene, and dried at room temperature. The amount of the resin was adjusted to 0.25 mass $\%$. To harden the resin after drying, the dried powder was heated to $473 \mathrm{~K}$ and kept for $7.2 \mathrm{ks}$ in air.

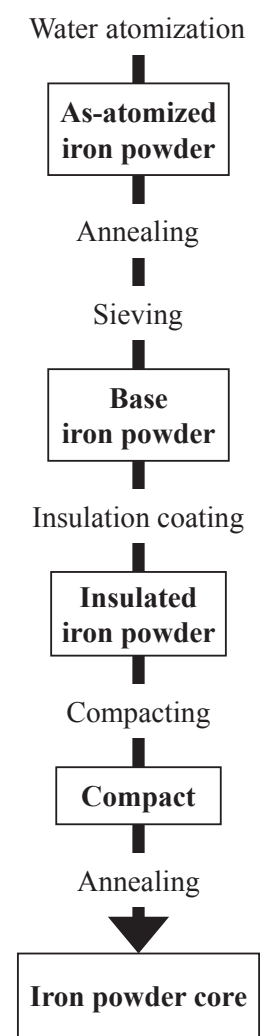

Fig. 1 Properties of the base iron powder. 
Table 1 Properties of the base iron powder.

\begin{tabular}{|c|c|c|c|c|c|c|}
\hline \multirow{2}{*}{$\begin{array}{c}\text { Apparent } \\
\text { Density } \\
/ \mathrm{Mg} \mathrm{m}^{-3}\end{array}$} & \multicolumn{6}{|c|}{ Particle size distribution $/ \%$} \\
\hline & $\begin{array}{c}180 \sim 150 \\
\mu \mathrm{m}\end{array}$ & $\begin{array}{c}150 \sim 106 \\
\mu \mathrm{m}\end{array}$ & $\begin{array}{c}106 \sim 75 \\
\mu \mathrm{m}\end{array}$ & $\begin{array}{c}75 \sim 63 \\
\mu \mathrm{m}\end{array}$ & $\begin{array}{c}63 \sim 45 \\
\mu \mathrm{m}\end{array}$ & $\leqq 45 \mu \mathrm{m}$ \\
\hline 3.57 & 0.1 & 23.5 & 32.8 & 8.4 & 17.2 & 18 \\
\hline
\end{tabular}

Table 2 Chemical composition.

$/ \operatorname{mass} \%$

\begin{tabular}{cccccccc}
\hline $\mathrm{C}$ & $\mathrm{Si}$ & $\mathrm{Mn}$ & $\mathrm{P}$ & $\mathrm{S}$ & $\mathrm{Al}$ & $\mathrm{N}$ & $\mathrm{O}$ \\
\hline 0.0043 & 0.017 & 0.04 & 0.009 & 0.0004 & 0.003 & 0.0005 & 0.0673 \\
\hline
\end{tabular}

The insulated iron powder was compacted into a ring shape with an outer diameter of $38 \mathrm{~mm}$, inner diameter of $25 \mathrm{~mm}$, and height of $6 \mathrm{~mm}$ at a pressure of $980 \mathrm{MPa}$ at room temperature. The compacts were annealed in nitrogen atmosphere under atmospheric pressure for $2.7 \mathrm{ks}$. The annealing temperature was varied from $673 \mathrm{~K}$ to $973 \mathrm{~K}$ to control the dislocation density. In the following, the powder cores annealed at $673 \mathrm{~K}, 823 \mathrm{~K}$ and $973 \mathrm{~K}$ will be denoted as P673, P823 and P973, respectively.

\subsection{Evaluation}

The direct current (DC) hysteresis loop was measured with a DC magnetometer (Metron, Inc. Type: SK-110). The magnetic path length, cross-sectional area and density were calculated from dimension and weight of the core. The magnetic path length was defined as the average of the outer and inner circumferences. Primary and secondary coils (100 and 40 turns, respectively) were wound with $\phi 0.6 \mathrm{~mm}$ insulated copper wire. The maximum magnetic induction was $1.0 \mathrm{~T}$, and the coercive force was evaluated from the DC hysteresis loop.

The dislocation density was estimated with the Williamson-Hall method ${ }^{6}$ ) from the full width at half maximum (FWHM) of the X-ray diffraction peak. The cross section perpendicular to the circumference was polished and etched with nital, and the FWHMs of (110), (211) and (220) planes for the etched surface were applied to the estimation of the dislocation density.

The same polished surface was observed with an optical microscope. An additional nital etching was applied to expose the grain boundaries. The observation area was $150 \times 210 \mu \mathrm{m}$, and the crystal grain size within the area was estimated with an intercept method. ${ }^{7)}$ The average over the four areas on a specimen was defined as the average crystal grain size, $d_{\mathrm{i}}$, of the specimen.

Crystal orientation of the same specimen was analyzed by Electron BackScattering Diffraction (EBSD) using a Scanning Electron Microscope (SEM). The microscopic dislocation density distribution was evaluated from the Kernel Average Misorientation (KAM) ${ }^{8)}$ and the Grain Orientation Spread $(\mathrm{GOS})^{8)}$ results. The analyzing area and step of the EBSD measurement were $500 \mu \mathrm{m} \times 500 \mu \mathrm{m}$ and $1 \mu \mathrm{m}$, respectively, and the kernel shape was a regular hexagon. The KAM value was calculated as the average misorientation angle between a kernel and the six neighboring ones, and the misorientation angles of $5^{\circ}$ or more were regarded as grain boundaries and excluded from the KAM calculation.

The GOS value shows the magnitude of the dislocation density accumulated in an individual crystal grain. In calculating the GOS value, a region surrounded by a boundary with a kernel misorientation of $5^{\circ}$ or more was regarded as one crystal grain. After calculating the average crystal orientation in a crystal grain, the misorientation between the orientations of the individual kernels in the grain and the average crystal orientation were calculated, and the average of those values was used as the GOS value.

KAM is an index of the dislocation density in each kernel of the EBSD analysis, and a larger KAM value indicates a larger dislocation density. ${ }^{8}$ ) GOS is an index of the dislocation density in each crystal grain; a larger GOS value indicates a larger dislocation density. ${ }^{8)}$

\section{Experimental Results}

The relationship between the annealing temperature and coercive force of the iron powder core is shown in Fig. 2. The coercive force decreases with an increase in the annealing temperature. The relationship between the annealing temperature and dislocation density is shown in Fig. 3. The dislocation density decreases with an increase in the annealing temperature. The relationship between the dislocation density and coercive force is shown Fig. 4. The coercive force decreases with a decrease in the dislocation density.

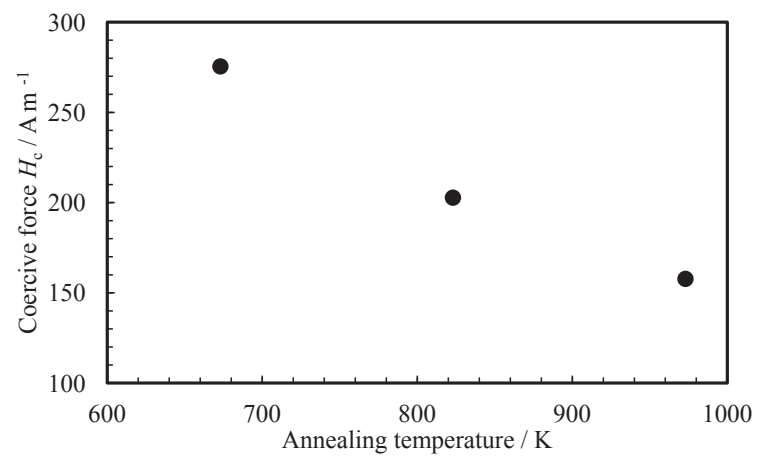

Fig. 2 Relationship between annealing temperature and coercive force of iron powder cores.

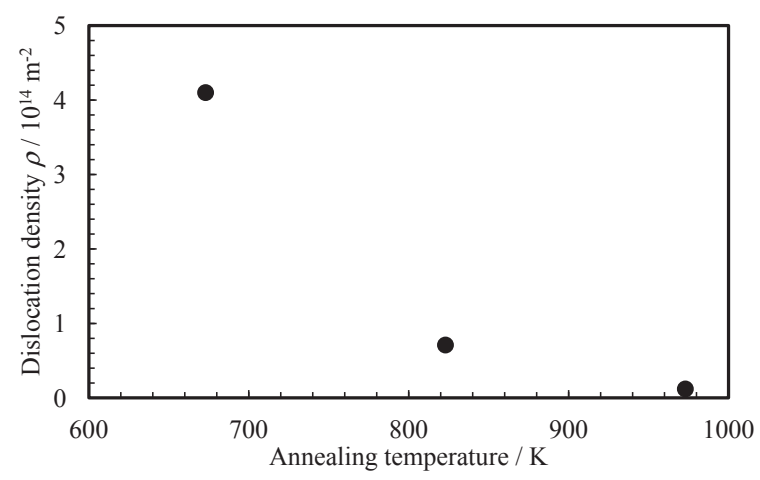

Fig. 3 Relationship between annealing temperature and dislocation density of iron powder cores. 


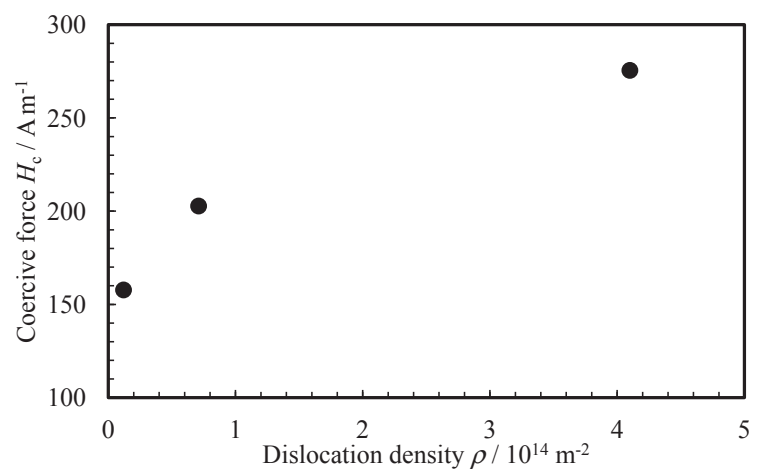

Fig. 4 Relationship between dislocation density and coercive force of iron powder cores.
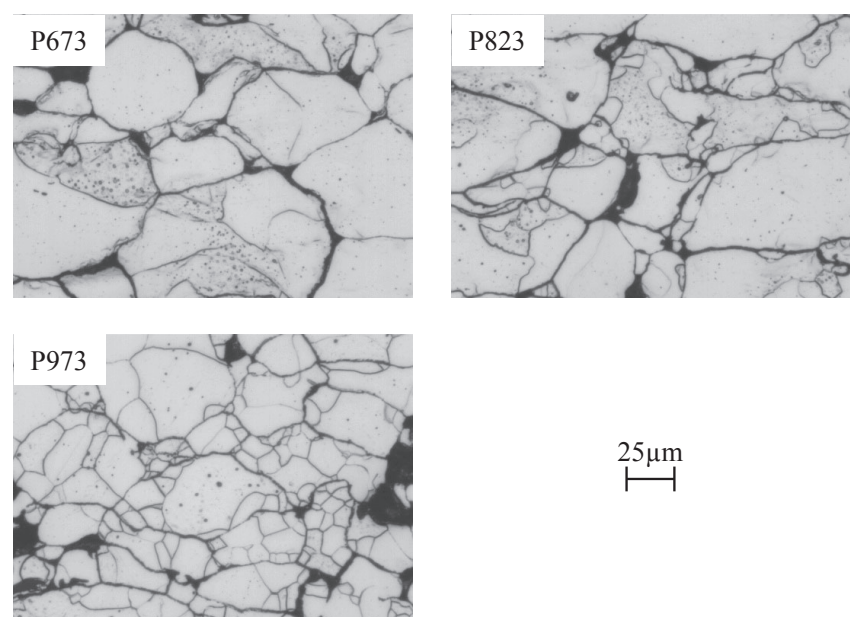

Fig. 5 Optical micrographs of the cross section of iron powder cores and steel sheet cores.

The microstructures of the iron powder cores are shown in Fig. 5. The grain boundaries within the iron powder particles are confirmed and crystal grain size decreases with an increase in annealing temperature. The relationship between

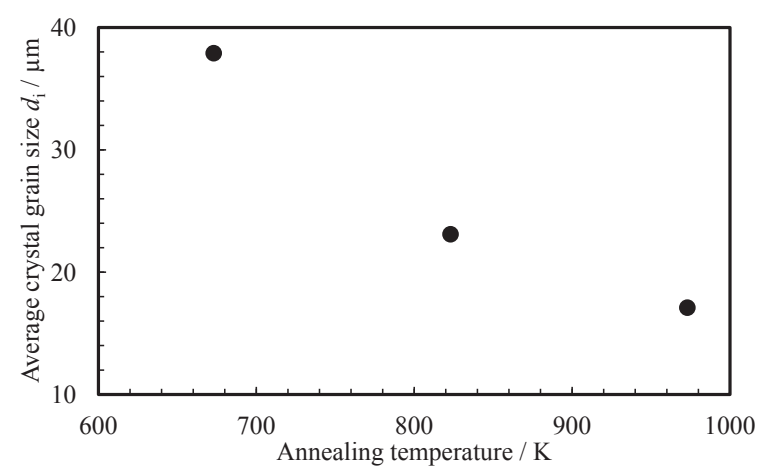

Fig. 6 Relationship between annealing temperature and crystal grain size of iron powder cores.

the annealing temperature and the average crystal grain size $d_{\mathrm{i}}$ measured by the intercept method from the optical micrographs is shown in Fig. 6. The $d_{\mathrm{i}}$ value decreases from $37.9 \mu \mathrm{m}$ to $17.1 \mu \mathrm{m}$ with an increase in the annealing temperature from 673 to $973 \mathrm{~K}$.

The KAM maps of the cross sections of the iron powder cores are shown in Fig. 7. The specimen P673 (annealing temperature: $673 \mathrm{~K}$ ) shows high KAM area larger than those annealed at higher temperatures. Furthermore, the KAM values in the vicinity of the iron powder particle surfaces are higher than those in the inner area of the particles. This indicates that the dislocation density in the vicinity of the particle surface is higher than those of the inner area of the particles. In P823 (annealing temperature: $823 \mathrm{~K}$ ), there are some areas where the KAM values are less than $1^{\circ}$, and in P973 (973 K), the KAM values are almost less than $1^{\circ}$ over most of the observed area.

The GOS maps of the cross sections of the iron powder cores are shown in Fig. 8. Although most of the crystal grains in P673 show GOS values of $6^{\circ}$ or higher, the number of crystal grains with GOS values of less than $6^{\circ}$ increases with an increase in the annealing temperature, and in P973, most of the crystal grains have GOS values of less than $6^{\circ}$.
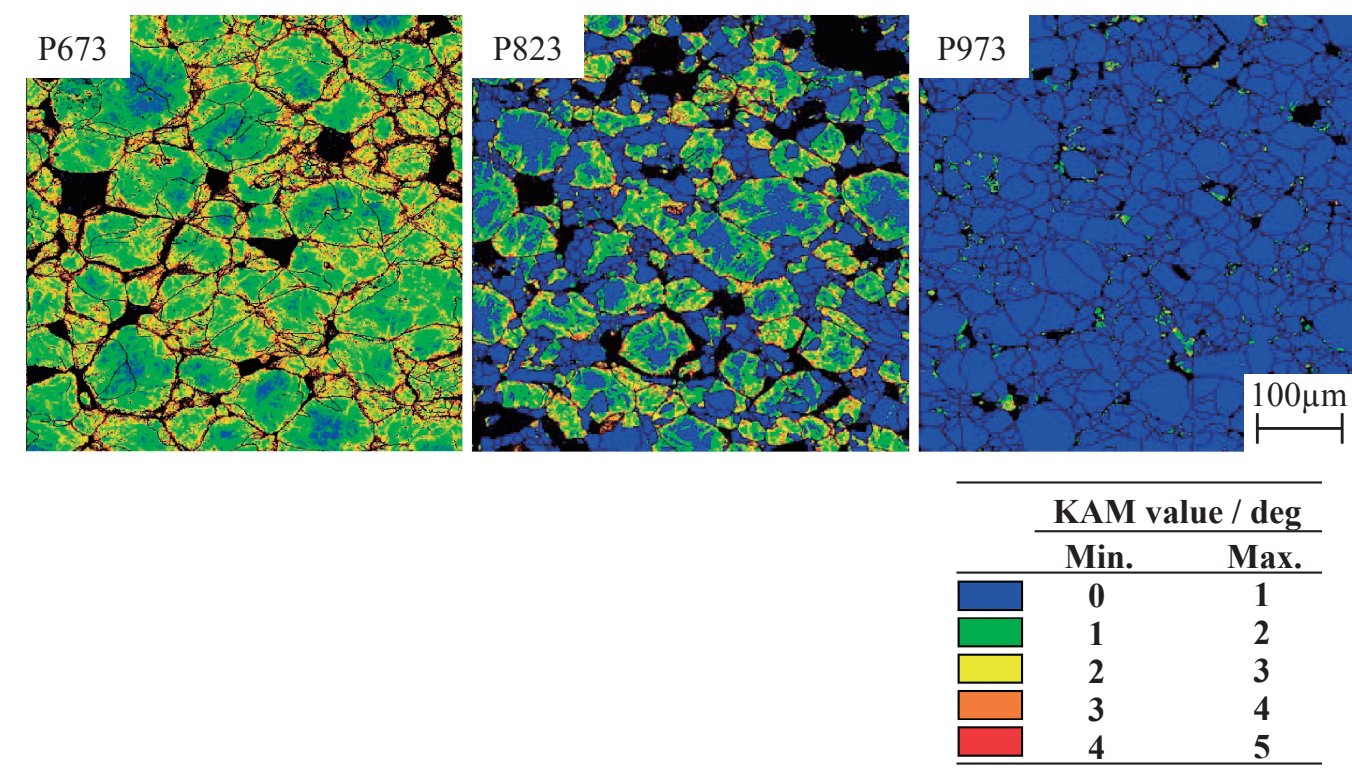

Fig. 7 KAM maps of the iron powder cores. The annealing temperature are $673 \mathrm{~K}(\mathrm{P} 1), 823 \mathrm{~K}(\mathrm{P} 2)$ and $973 \mathrm{~K}(\mathrm{P} 3)$. 

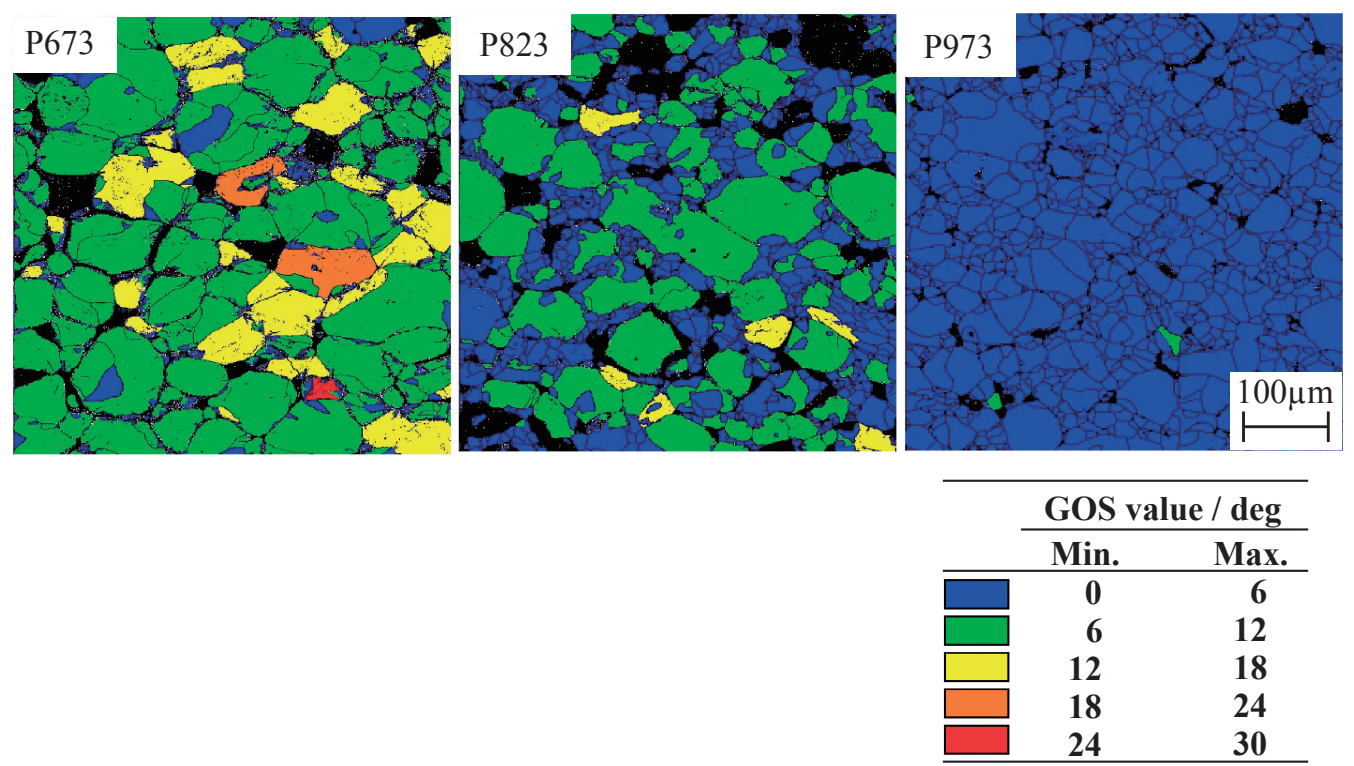

Fig. 8 GOS maps of the iron powder cores. The annealing temperature are $673 \mathrm{~K}(\mathrm{P} 1), 823 \mathrm{~K}(\mathrm{P} 2)$ and $973 \mathrm{~K}(\mathrm{P} 3)$.

\section{Discussion}

\subsection{Influence of plastic deformation on microstructure of iron powder cores}

As described above, the dislocation density and the average crystal grain size decreases with an increase in the annealing temperature (Fig. 2 and Fig. 3). In connection with such phenomena, Takashita et al. ${ }^{3)}$ have proposed a mechanism of crystal grain size refinement in terms of recrystallization; the dislocations induced trough the compaction process act as the driving force for recrystallization during the annealing. In this section, the mechanism is discussed in detail based on the KAM and GOS values.

As mentioned above, the GOS value is an index showing the crystal orientation distribution within a crystal grain. The GOS value increases with an increase in the crystal orientation distribution in the grain, hence an increase in the GOS value indicates an increase in the dislocation density in the grain. ${ }^{8)}$ Recrystallized grains are newly formed in the high dislocation density area during annealing, and they have reduced dislocation densites. ${ }^{9)}$ Therefore, the GOS value of recrystallized grains is lower than that of grains deformed by the compaction.

The GOS frequency distributions from the GOS map in Fig. 8 is shown in Fig. 9. Some small peaks are observed in the range from $4^{\circ}$ to $18^{\circ}$ for the specimen annealed at $673 \mathrm{~K}$. For the $823 \mathrm{~K}$ annealed specimen, however, a higher peak appears on the lower angle side, and for $973 \mathrm{~K}$ annealing, peaks on higher angle side disappear and only one peak remains at approximately $1^{\circ}$. The fact that the KAM value of P973 are less than $1^{\circ}$ over most of the observed area (Fig. 7) indicates that the peak is caused by recrystallized grains.

One the assumption that the higher edge of the peak in P973 (GOS value: $2.5^{\circ}$ ) represents the threshold of recrystallization, the crystal grains on the GOS map are separated into recrystallized grain $\left(\mathrm{GOS}<2.5^{\circ}\right)$ and unrecrystallized $\left(\mathrm{GOS} \geqq 2.5^{\circ}\right)$ area as shown in Fig. 10 . The relationship between the annealing temperature and area

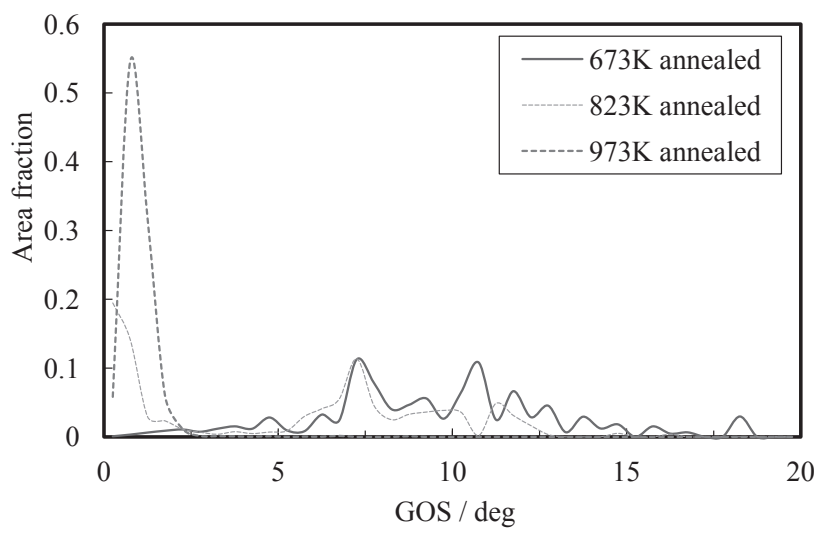

Fig. 9 Area fraction of GOS value.

fraction of the recrystallized grains to the total EBSD observation area are shown in Fig. 11. In Fig. 10, the particle surfaces of some of the grains are denoted by red lines. In P673, the area fraction of the recrystallized grain is as small as 0.04 . Small amount of recrystallized nucleus, however, are observed near the surfaces of some of the particles as shown by the green arrows. In P823, many recrystallized grains are observed in the vicinity of the particle surface, and in P973, the recrystallized grains become dominant.

On the other hand, in the KAM map of P673 in Fig. 7, regions with higher KAM values concentrate along the particle surface. Recrystallized grains are newly formed in the high dislocation density area and grow by the strain energy of dislocation. ${ }^{9)}$ Therefore, from Fig. 7 and Fig. 10, nucleation of recrystallized grains by annealing occurs in the vicinity of the particle surface, where a large number of dislocations are introduced during compaction, and the recrystallized grain growth proceeds by the strain energy of dislocation. These results support the mechanism proposed by Takashita et al. ${ }^{3)}$

Next, we discuss the growth of the recrystallized grain during annealing process. The equivalent circle diameter $d_{\mathrm{c}}$ of a crystal grain is given as 

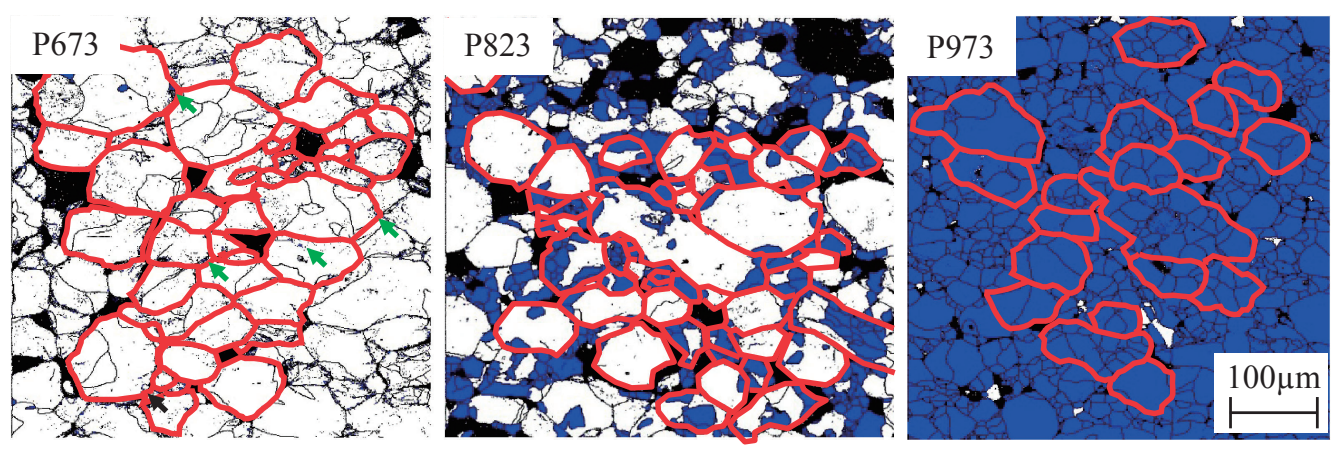

Particle surface

Recrystallized grain

Non recrystallized grain

Fig. 10 GOS maps of the iron powder cores (Blue area is recrystallized grain).

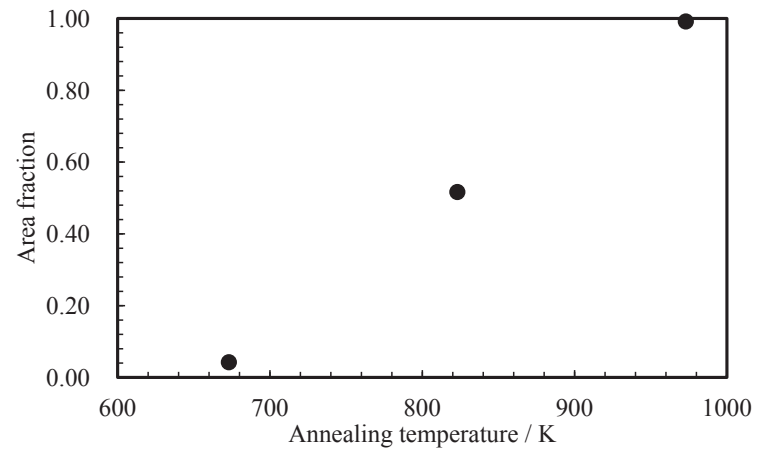

Fig. 11 Relationship between annealing temperature and area fraction of recrystallized grain.

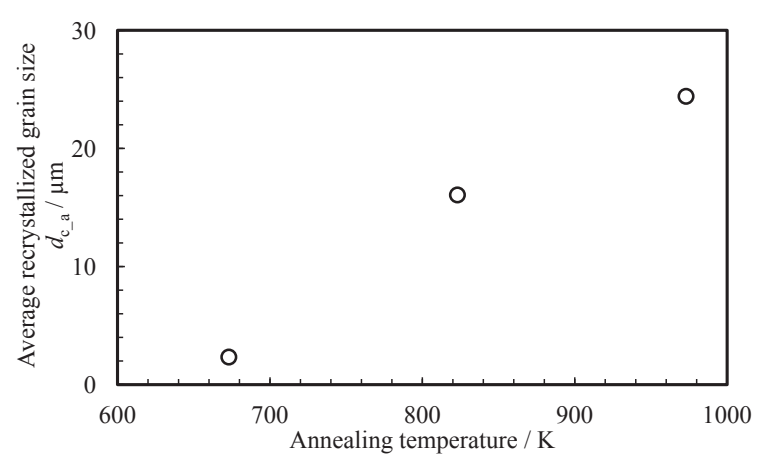

Fig. 12 Relationship between annealing temperature and average recrystallized grain size.

$$
d_{\mathrm{c}}=2 \sqrt{A / \pi},
$$

where $A$ is the area of the crystal grain, and calculated from the size and number of the EBSD kernels included in the grain. The accumulated area fraction, in which the area of the recrystallized grains are accumulated from the small $d_{\mathrm{c}}$ side, is also calculated as a function of $d_{c}$, and the point where this accumulated area fraction reaches $50 \%$ of the total area fraction of recrystallized grains is defined as the average diameter of recrystallized grain $d_{\mathrm{c}_{\mathrm{a}} \mathrm{a}}$. The relationship between annealing temperature and $d_{\text {c_a }}$ of the recrystallized grains is

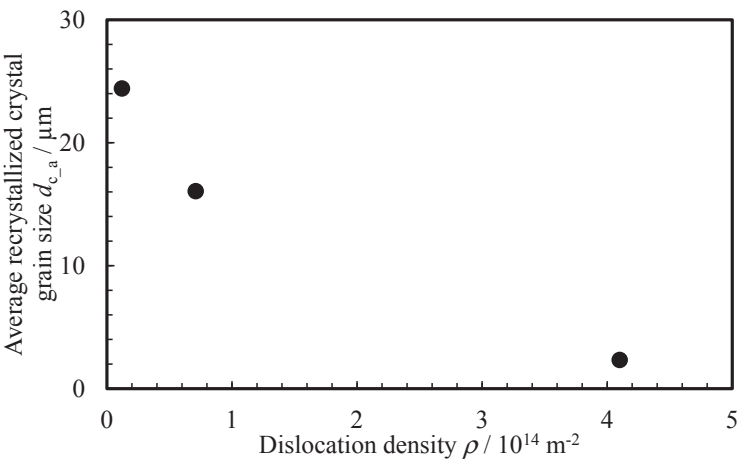

Fig. 13 Relationship between dislocation density and average recrystallized grain size.

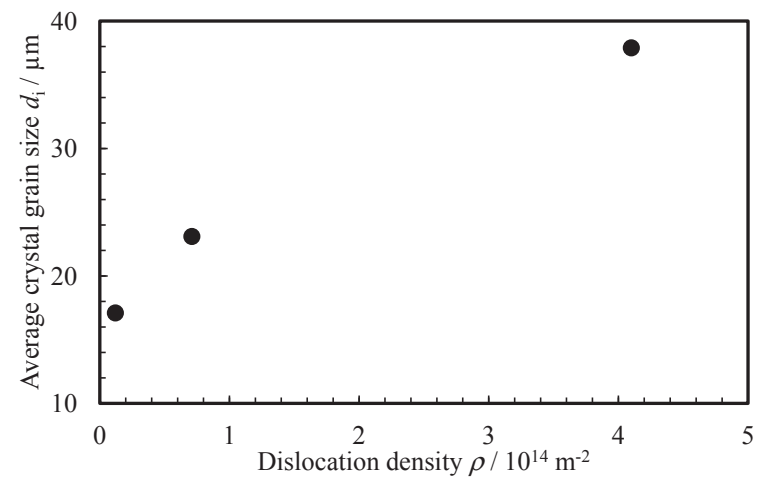

Fig. 14 Relationship between dislocation density and average crystal grain size measured with intercept method.

shown in Fig. 12. The $d_{\text {c_a }_{-}}$value increases with an increase in the annealing temperature.

The relationship between the dislocation density $\rho$ and the average recrystallized grain size $d_{\mathrm{c} \_a}$ is shown in Fig. 13. The $d_{\mathrm{c} \_\mathrm{a}}$ value increases with a decrease in the dislocation density. This indicates that the strain energy due to dislocations in the unrecrystallized grains acts as the driving force of the growth of recrystallized grains.

The relationship between the dislocation density $\rho$ and $d_{\mathrm{i}}$ obtained by the intercept method is shown in Fig. 14. The $d_{\mathrm{i}}$ decreases with a decrease in dislocation density in spite that 
$d_{\text {c_a }}$ increases with a decrease in the dislocation density. The $d_{\mathrm{i}}$ value is the average of the whole grains including recrystallized and unrecrystallized grains. Accordingly, during annealing, $d_{\mathrm{i}}$ decreases as the recrystallized grains are created because the recrystallized grains are much finer than the unrecrystallized grains, and the area fraction of the fine recrystallized grains increases with an increase in annealing temperature.

From the discussion above, the iron powder cores include unrecrystallized grains with high dislocation densities and recrystallized grains with low dislocation densities, and the fraction of unrecrystallized grains decreases with an increase in the annealing temperature (Fig. 8). This causes the change in the dislocation density shown in Fig. 3.

\subsection{Influence of plastic deformation on coercive force of iron powder cores}

As described above, the average crystal grain size $d_{\mathrm{i}}$ and the dislocation density $\rho$ change at the same time. In the discussion bellow, we try to separate the influences of the dislocation density and grain size on coercive force.

The coercive force $H_{\mathrm{c}}$ of a magnetic material depends on the magnetocrystalline anisotropy and the number of domain wall pinning sites such as grain boundaries, inclusions and dislocations. Pfeifer et al. ${ }^{10)}$ have analyzed the coercive force as the sum of the contributions of such pinning mechanisms, and expressed as eq. (2)

$$
H_{\mathrm{c}}=H_{\mathrm{ck}}+H_{\mathrm{c} \_ \text {dis }}+H_{\text {c_ex }}
$$

where, $H_{\text {ck }}, H_{\text {c_dis }}$ and $H_{\text {c_ex }}$ are the contributions from crystal grain boundaries, dislocations and the others, respectively. Previous studies on $\mathrm{Ni}-\mathrm{Fe},{ }^{10)} \mathrm{Fe}-\mathrm{Si}^{11)}$ and $\mathrm{Co}-\mathrm{Fe}^{12)}$ have shown a linear relationship between $H_{\mathrm{ck}}$ and the reciprocal of the crystal grain size. Takashita et al. ${ }^{13)}$ have shown a relationship between the average crystal grain size $d$ and the coercive force for pure iron powder cores and pure iron sheets as

$$
H_{\mathrm{ck}}=1.3 \times 10^{3} / d
$$

On the other hand, Yaegashi et al. ${ }^{5)}$ have given the contribution of the dislocation density $\rho$ to the coercive force in a polycrystalline bulk iron as

$$
H_{\mathrm{c} \_ \text {dis }}=\gamma \cdot \rho^{1 / 2}
$$

where $\gamma$ is a constant depending on the saturation magnetic flux density, area of magnetic domain walls per unit volume, mean spacing of the domain walls, and distribution of dislocations. Substituting eq. (3) and eq. (4) into eq. (2), we obtain an expression for the coercive force as

$$
H_{\mathrm{c}}=1.3 \times 10^{3} \cdot d^{-1}+\gamma \cdot \rho^{1 / 2}+H_{\mathrm{c} \_\mathrm{ex}}
$$

here, $d$ in eq. (3) is equivalent to the average crystal grain size $d_{\mathrm{i}}$ in this study. Equation (5) is converted into the form of

$$
H_{\mathrm{c}}-1.3 \times 10^{3} \cdot d_{\mathrm{i}}^{-1}=\gamma \cdot \rho^{1 / 2}+H_{\mathrm{c} \_\mathrm{ex}},
$$

Under an assumption that $H_{\text {c_ex }}$ is constant, the constants $\gamma$ and $H_{\mathrm{c} \_ \text {ex }}$ are estimated from the experimental results of $H_{\mathrm{c}}, d_{\mathrm{i}}$ and $\rho$. Actually, the assumption is reasonable in this study, because the causes of $H_{\text {c_ex }}$, such as the inclusions ${ }^{4)}$ and void

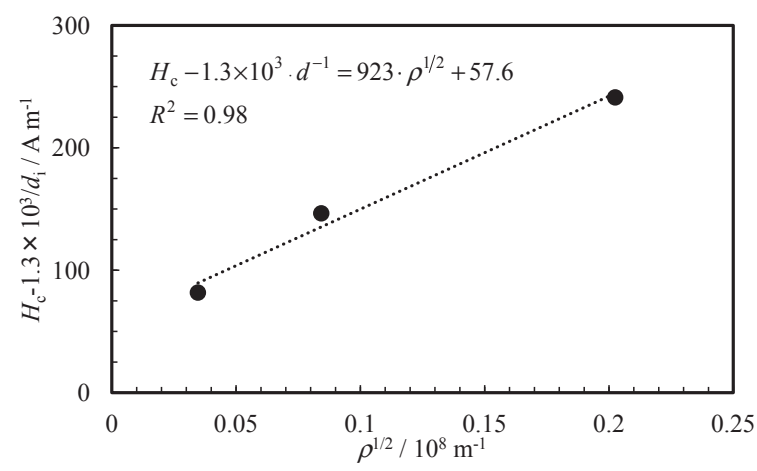

Fig. 15 Relationship between $\rho^{1 / 2}$ and $H_{\mathrm{c}}-1.3 \times 10^{3} d_{\mathrm{i}}^{-1}$ of the iron powder cores.

space between particles $^{13)}$ are identical for all of the specimens prepared from the same raw material prepared through the same compaction process and conditions.

The value of $H_{\mathrm{c}}-1.3 \times 10^{-3} / d_{\mathrm{i}}$ calculated from the experimental results are plotted against $\rho$ in Fig. 15. This shows a linear relationship with a regression of

$$
H_{\mathrm{c}}-1.3 \times 10^{3} \cdot d_{\mathrm{i}}^{-1}=923 \cdot \rho^{1 / 2}+57.6 .
$$

As described above, the iron powder cores in this study consist of unrecrystallized and recrystallized grains. Accordingly, the distribution of dislocations is also heterogeneous, i.e. the dislocations segregate into the unrecrystallized grains. However, the coercive force of the iron powder cores evaluated in this study is in good agreement with the model of eq. (6) with the average crystal grain size $d_{\mathrm{i}}$ including both of the unrecrystallized and recrystallized grains, and the dislocation density $\rho$ averaged over the heterogeneous distribution. Based on this fact, the relationship between the microstructure and coercive force can be explained quantitatively by using boundary pinning model, even in the case of an iron powder core with the heterogeneous distribution of dislocations in each crystal grain.

The $H_{\text {c_ex }}$ value is estimated as $57.6 \mathrm{~A} / \mathrm{m}$ from the regression analysis based on eq. (7). This is regarded as the contributions from inclusions and interparticle space. These two contributions are not separated for now. Experiments with varying cores density is planned as a future work to control the inter particle spacing and to evaluate these two contributions separately.

The $\gamma$ value of 923 from the regression analysis is higher than the value of 645 reported by Yaegashi et al..$^{5)}$ Difference in the dislocation distribution in the crystal grains is a possible cause of the difference in $\gamma$. Yaegashi et al. inferred that the reason why the $\gamma$ value of a JIS standard SFVQ (steel forging vessel and quenched) commercial steel is higher than that of polycrystalline iron is that the number of the dislocation cell walls with a low angle grain boundary form is larger. In addition, Träuble et al. ${ }^{14)}$ have shown theoretically that the $\gamma$ value is enhanced by clustering of dislocation. From these previous studies, ${ }^{5,14)}$ it can be inferred that the iron powder cores in this study would include dislocation structures like clusters or cell walls, resulting in an enhancement of $\gamma$ value. In order to verify this hypothesis, observation of the cell walls by TEM will be necessary in future research. 


\section{Conclusions}

In this research, the influence of the dislocation density on the coercive force of pure iron powder cores was evaluated by separating the contributions of the crystal grain size and inclusions. The conclusion is as follow;

(1) The average dislocation density and average crystal grain size in the iron powder cores decreased with an increase in annealing temperature.

(2) By GOS mapping derived by the SEM/EBSD method, it was revealed that the iron powder cores have a heterogeneous microstructure comprising unrecrystallized grains with high dislocation densities and recrystallized grains with low dislocation densities.

(3) The area fraction of recrystallized grains in the observation area increased with an increase in annealing temperature. The decrease in the average dislocation density mentioned in (1) is considered to be due to an increase in the area fraction of recrystallized grains.

(4) Furthermore, the crystal grain size of the recrystallized grains was smaller than that of the unrecrystallized grains. It indecates that the crystal grain size decreased due to an increase in the area fraction of recrystallized grains an increase in annealing temperature.

(5) On the other hand, if the average crystal grain size and the average dislocation density including both recrystallized grains and unrecrystallized grains is used, the behavior of the coercive force is consistent with the classical magnetic domain wall pinning model.

(6) That is, coercive force is the sum of the contributions of all of the factors that increase coercive force. Specifically, the coercive force attributable to crystal grains boundaries is proportional to the reciprocal of the average crystal grain size, and the coercive force attributable to dislocation density is proportional to the square root of the average dislocation density.

(7) Accordingly, it was shown that the relationship between the microstructure and coercive force of iron powder cores can be explained quantitatively by using the magnetic domain wall pinning model, even if the heterogeneous microstructure in which the dislocation density is different in each crystal grains.

\section{REFERENCES}

1) M. Sugiyama, T. Yamaguchi, T. Ohkouchi, H. Kishimoto, T. Hattori and T. Saito: SOKEIZAI 51 (2010) 24-29.

2) T. Tokuoka, T. Maeda and T. Ishimine: SOKEIZAI 52 (2011) 11-18.

3) T. Takashita, N. Naomichi and Y. Ozaki: Mater. Trans. 57 (2016) 18591867.

4) K. Nishi, Y. Aono, K. Okamoto, T. Imagawa and K. Souma: J. Jpn. Soc. Powder Metallurgy 63 (2016) 63-69.

5) K. Yaegashi: Tetsu-to-Hagané 91 (2005) 655-661.

6) R.L. Fullman: Trans. AIME 197 (1953) 447-452.

7) K. Nakashima, Y. Nimura, H. Hidaka, S. Tsuchiyama and S. Takaki: CAMP-ISIJ 17 (2004) 396-399.

8) K. Nomura, K. Kubusiro, S. Takahashi and H. Yoshizawa: J. Soc. Mater. Sci. Jpn. 61 (2012) 371-376.

9) E. Furubayashi: Saikessyoutozairyousoshiki, (Uchidarokakuho, 2000) p. 7.

10) F. Pfeifer and C. Radeloff: J. Magn. Magn. Mater. 19 (1980) 190-207.

11) G. Herzer: IEEE Trans. Magn. 26 (1990) 1397-1402.

12) R.H. Yu, S. Basu, Y. Zhang, A. Parvizi-Majidi and J.Q. Xiao: J. Appl. Phys. 85 (1999) 6655-6659.

13) T. Takashita, N. Naomichi and Y. Ozaki: Proc. of PM2016, (2016).

14) H. Träuble: Magnetism and Metallurgy, ed. by A.E. Berkowitz and E. Kneller, (Academic Press, 1969) pp. 662-666. 